\title{
Morfología de la lavandera cascadeña Motacilla cinerea L., 1758 en Gipuzkoa (región cantábrica).
}

\section{Morphology of the grey wagtail Motacilla cinerea L., 1758 in Gipuzkoa (Cantabrian region).}

Nerea Pagaldai ${ }^{1}$, José I. Jauregi ${ }^{1}$, Irati Zubia ${ }^{1}$, Iñaki Aranguren ${ }^{1}$, Javier Goikoetxea ${ }^{1}$, José M. Sánchez ${ }^{1}$, Juan Arizaga ${ }^{1 *}$

\section{Resumen}

El objetivo de este artículo es describir la morfología de la lavandera cascadeña Motacilla cinerea L., 1758 (y en particular su variación según sexos y clases de edad) en Gipuzkoa. Además, y dado que en las aves de primer año no es posible conocer el sexo a partir de la coloración, se realiza un Análisis Discriminante (AD) con el fin de ofrecer una fórmula biométrica para la diferenciación de sexos en aves no adultas. La morfología de la lavandera cascadeña en el área de estudio varía tanto entre sexos como entre edades. El sexo tuvo un efecto más fuerte que la edad, al influir en cuatro variables (longitud alar, apuntamiento del ala, longitud de la cola y cráneo+pico) frente a las tres de la edad (las cuatro de antes menos el apuntamiento del ala). Solamente el tarso no varió ni entre sexos ni entre edades. En general, los valores más bajos se hallaron en hembras jóvenes y, los más altos, en machos adultos. El análisis discriminante proporcionó una fórmula que sólo clasificó bien el sexo en el $69 \%$ de la muestra de jóvenes lavanderas cascadeñas. Esto es debido a la existencia de un alto solapamiento biométrico entre los sexos, dentro de esta edad, lo que en la práctica supone la no recomendación del uso de la ecuación dada su baja fiabilidad.

Palabras clave: anillamiento, biometría, aves, edad, ríos, sexo.

1 Sociedad de Ciencias Aranzadi. Departamento de Ornitología. Zorroagagaina, 11. 20014 Donostia-San Sebastián (Spain). 


\begin{abstract}
The aim of this study was to describe the morphology of a population of grey wagtails Motacilla cinerea L., 1758, breeding in Gipuzkoa (Spain). Additionally, due to the difficulty involved in sexing first-year birds based on their colouration, we carried out Discriminant Analysis (DA) to find an equation that would allow us to determine the sex of individuals using their morphological data. The morphology varied both between ages and sexes. Sex had a greater effect than age, as it varied in four variables (wing length, wing pointedness, tail length, head+bill length), whilst age only varied in three of these variables (all the aforementioned, except wing pointedness). Overall, the lowest values were found in young females, whereas the highest ones were typical of adult males. The DA provided an equation that enabled us to classify correctly only $69 \%$ of the sample and, therefore, its application for the reliable sexing of young birds in the field is not recommended.
\end{abstract}

Key words: ringing, biometrics, birds, age, rivers, sex.

\title{
Laburpena
}

Artikulu honen xedea Motacilla cinerea L., 1758 buztanikara horiaren Gipuzkoako populazioak zer morfologia duen deskribatzea da (zehazki, sexu eta adin taldeen araberako aldakortasuna). Gainera, lehen urteko banakoetan kolorazio bidez sexua antzematerik ez dagoenez, Analisi Diskriminatzaile (AD) bat burutu da, hegazti ez-helduetan sexua bereizteko formula biometriko bat lortzeko asmoz. Ikerketa eremuko buztanikara horiaren morfologia sexuaren zein adinaren arabera aldatzen da. Sexuak adinak baino eragin handiagoa izan zuen, lehenak lau aldagaietan eraginez (hegoen luzera, zorroztazuna, buztanaren luzera eta burezurra+mokoa); eta bigarrenak, aldiz, hiru aldagaietan (aipatutako laurak hegoen zorroztazuna izan ezik). Tartsoa izan zen ez sexu ezta adin talde artean aldaketarik jasan ez zuen aldagai bakarra. Eme gazteetan behatu ziren orokorrean baliorik baxuenak; altuenak, aldiz, ar helduetan. Analisi diskriminatzaileak emandako formulak buztanikara hori gazteen laginaren \%69 soilik sailkatu zuen modu egokian. Hori da banako gazteetan izaten den sexuen arteko biometria gainezartzen delako; beraz, ekuazioaren fidagarritasun eskasa dela-eta ez.

Gako hitzak: eraztunketa, biometria, hegaztiak, adina, errekak, sexua. 


\section{Introducción}

El análisis de la morfología constituye un capítulo esencial a la hora de describir especies, poblaciones o individuos, e.g. de distinta edad o sexo dentro de poblaciones, o para relacionar la morfología con diferentes aspectos ecológicos, como el ambiente o el comportamiento.

La lavandera cascadeña Motacilla cinerea L., 1758 es un paseriforme de amplia distribución paleártica, presente desde el oeste de Europa hasta el este de Asia. En España, se localiza en cursos dulceacuícolas, tanto en el norte, donde es común, como en el área mediterránea, donde ocupa zonas de montaña. En Gipuzkoa es ubicua, tanto en zonas montañosas como en la costa, donde se asocia a ríos como el Oria, Deba, etc.

Hasta la actualidad, la morfología de la lavandera cascadeña en España no ha sido descrita más que en Canarias, y no detalladamente. De un conjunto de variables (longitud del ala, cola y pico), sólo el ala parece que varía entre sexos, siendo más larga en machos. En este caso, no obstante, no se hace referencia al efecto de la edad en la morfología, aun siendo una variable que, probablemente, pueda tener una clara influencia sobre la misma .

El objetivo de este artículo es describir la morfología de la lavandera cascadeña (y en particular su variación según sexos y clases de edad) en Gipuzkoa (región cantábrica), donde se ubica uno de los principales núcleos poblacionales de España. Se espera que los machos sean mayores que las hembras y los adultos que los jóvenes. Además, y dado que en las aves de primer año no es posible conocer el sexo a partir de la coloración, se proporciona el resultado de un Análisis Discriminante (AD) con el fin de ofrecer una fórmula biométrica para la diferenciación de sexos en aves no adultas.

\section{Material y métodos}

\section{Área de muestreo y protocolo para la toma de muestras}

Se capturaron lavanderas cascadeñas durante un periodo de once años consecutivos (2008-2018), a lo largo de todo el ciclo anual, en 21 ríos de Gipuzkoa, pertenecientes a las cuencas del: Oiartzun, Urumea y Oria, todos ellos en la región cantábrica oriental. Se trata de una zona con gran cantidad de precipitaciones (media anual en Gipuzkoa: $1400 \mathrm{~mm}$ ) y temperaturas suaves $\left(14^{\circ} \mathrm{C}\right.$ de media anual). Los ríos tienen cursos cortos (el Oria, que es el más largo, tiene sólo 80 km de longitud) y caudales estables y cuantiosos, dada la alta pluviometría. La orografía de la provincia es accidentada.

Para la captura se emplearon redes de niebla (de $16 \mathrm{~mm}$ de luz de malla) situadas perpendicularmente al eje del río, de tal modo que las aves eran capturadas en el momento de moverse a lo largo del cauce. 
Cada individuo capturado se anilló y determinó su edad (jóvenes, edad EURING 3 o 5; ejemplar con un año calendario de vida; adultos, edad EURING 4 ó 6, ejemplar con dos o más años de vida), mediante el examen de las plumas del ala y cola, principalmente. La identificación de la edad se basa en la estrategia de muda de adultos y jóvenes, que es diferente. Mientras que los primeros llevan a cabo una muda completa (esto es, tras la cría renuevan todo su plumaje), los segundos realizan una muda parcial tras el periodo estival (esto es, previamente a su primer invierno). Concretamente, a diferencia de un adulto, un subadulto (aquí determinado como "joven") mantiene cierto número de plumas del ala y cola sin cambiar, esto es, retienen plumas de su plumaje juvenil. Estas plumas son generalmente más cortas que en los adultos y su color y forma es, también, diferente, lo que en conjunto permite distinguir las dos edades.

El sexo en adultos se determinó a través de su coloración, dada la existencia de dicromatismo sexual. En jóvenes, no obstante, la coloración no varía marcadamente entre sexos, de tal modo que su diferenciación es difícil (normalmente, imposible). En estos casos, el sexo se estableció sólo en los ejemplares que fueron recapturados como adultos, una vez han desarrollado una muda completa que les proporciona la coloración típica de adulto.

Finalmente, se midieron a cada ejemplar la longitud del ala, cola $( \pm 0,5 \mathrm{~mm})$, primarias P2 a P10 (numeración ascendente; $\pm 0,5 \mathrm{~mm})$, tarso $( \pm 0,1 \mathrm{~mm})$ y cráneo+pico $( \pm 0,1$ $\mathrm{mm}$ ). Además, se añadió aquí otra variable, la morfología del ala, para lo cual se calculó el siguiente índice: $\mathrm{M}=\left(\mathrm{P}_{\max }-\mathrm{P}_{10}\right)$ /ala, donde: $\mathrm{P}_{\max }=$ longitud de la primaria más larga, $\mathrm{P}_{10}=$ longitud de la primaria P10, ala = longitud del ala. Tanto la longitud alar como la de P2 a P10 se tomaron siempre en el ala derecha. Todas las medidas fueron tomadas por dos observadores (J. I. Jauregi, I. Aranguren), que con anterioridad al inicio de este proyecto se sometieron a un proceso de estandarización.

\section{Análisis estadísticos}

Primeramente, y puesto que contábamos con tamaños muestrales elevados, sólo se tuvieron en cuenta aquellos ejemplares para los que se habían medido todas las variables que se consideraron en este artículo y para los que se había determinado tanto su edad como su sexo. Además, no se utilizaron las recapturas para evitar pseudoreplicaciones. Así, la muestra inicial de 1611 casos se quedó en 432. Antes de comenzar con los análisis, además, todos los datos fueron examinados con el fin de eliminar posibles errores tanto durante la medición como a la hora de su digitalización. Para ello, se obtuvo para cada variable un diagrama de caja o "boxplot", de tal modo que los valores que claramente eran errores se eliminaron. Así, fueron omitidos del análisis un ejemplar con una longitud del tarso mayor que $30 \mathrm{~mm}$, dos ejemplares con 
cráneo+pico $>36 \mathrm{~mm}$ y otro ejemplar con cola $<80 \mathrm{~mm}$. El tamaño muestral definitivo, en consecuencia, se quedó en 428 ejemplares.

Cada una de las variables mostró un patrón de distribución normal (test de normalidad Kruskal-Wallis; $P>0,05)$. Se desarrolló un ANOVA de K muestras con cada una de las variables morfológicas, considerando como factores de clasificación la edad, sexo y periodo. El periodo se incluyó para determinar si la morfología varía entre la época en que la probabilidad de capturar aves de origen extranjero (esto es, aves en paso migratorio y/o invernantes) es baja o alta. Para ello, las capturas que se obtuvieron desde octubre hasta marzo se incluyeron en el periodo "invierno", cuando esta probabilidad es alta, mientras que las que se obtuvieron desde abril hasta septiembre se incluyeron en el periodo "verano", cuando esta probabilidad es baja y, en consecuencia, es correcto asumir que las aves capturadas pertenecen con una alta probabilidad a la población local, sensu lato. Como prueba a posteriori se empleó un test de Tukey-B, dada la homogeneidad de varianza ( $F_{\text {Levene, }} P>0,05$ en todos los casos).

Por otro lado, para comparar la morfología de la muestra de Gipuzkoa con la de otras zonas se llevó a cabo un test de t para 1 muestra para cada una de las variables morfológicas, donde el valor de referencia fue el que se obtuvo a través de la literatura. Este test se repitió para toda la muestra y para la fracción de aves capturadas en verano.

Finalmente, se aplicó un Análisis Discriminante $(A D)$ para obtener una fórmula que permitiera determinar el sexo a partir de la morfología en jóvenes. En este AD se incluyeron sólo las variables que variaron entre sexos (para la submuestra de aves jóvenes): longitud alar, cola y cráneo+pico. Se omitieron por tanto el tarso así como el apuntamiento del ala y la cola (efecto sexo: P > 0,05; detalles estadísticos no mostrados). Para llevar a cabo el AD se utilizó la función "Ida" del paquete "MASS" para R. El AD se desarrolló considerando el $60 \%$ de la submuestra; el restante $40 \%$ se empleó para validar la función discriminante.

\section{Resultados}

En conjunto, se estudió una muestra de 428 ejemplares (para más detalles ver Tabla 1), repartidos en 230 ejemplares en verano y 198 en invierno.

La morfología de la lavandera cascadeña en el área de estudio varía tanto entre sexos como entre edades (Tabla 2). El sexo tuvo un efecto más fuerte que la edad, al influir en cuatro variables frente a las tres de la edad. La ausencia de interacción sexo-edad permitiría concluir sobre efectos de cada factor de manera independiente. En general, los valores más bajos se hallaron en hembras jóvenes y, los más altos, en machos adultos (Tabla 1 y 3). Por otro lado, el periodo sólo influyó en la longitud alar, si bien en el 


\begin{tabular}{|l|c|c|c|c|}
\hline & \multicolumn{2}{|c|}{ Jóvenes } & \multicolumn{2}{c|}{ Adultos } \\
\hline Verano & $\sigma^{\prime}(n=57)$ & $q(n=56)$ & $\sigma^{\prime}(n=66)$ & $q(n=51)$ \\
\hline Longitud alar & $83,3 \pm 1,9$ & $81,6 \pm 1,8$ & $84,2 \pm 2,2$ & $82,4 \pm 1,7$ \\
\hline Apuntamiento del ala & $0,20 \pm 0,01$ & $0,20 \pm 0,01$ & $0,21 \pm 0,02$ & $0,20 \pm 0,01$ \\
\hline Cola & $95,7 \pm 4,0$ & $94,9 \pm 3,6$ & $96,5 \pm 3,9$ & $95,1 \pm 3,9$ \\
\hline Tarso & $21,4 \pm 0,7$ & $21,3 \pm 0,8$ & $21,3 \pm 0,8$ & $21,4 \pm 0,9$ \\
\hline Cráneo+Pico & $32,7 \pm 0,6$ & $32,4 \pm 0,6$ & $32,6 \pm 0,6$ & $32,5 \pm 0,7$ \\
\hline Invierno & $\sigma^{\prime}(n=72)$ & $\wp(n=24)$ & $\sigma^{\prime}(n=81)$ & $\wp(n=21)$ \\
\hline Longitud alar & $83,5 \pm 1,7$ & $81,7 \pm 1,5$ & $84,9 \pm 1,8$ & $82,5 \pm 1,6$ \\
\hline Apuntamiento del ala & $0,20 \pm 0,01$ & $0,20 \pm 0,02$ & $0,21 \pm 0,01$ & $0,20 \pm 0,01$ \\
\hline Cola & $96,7 \pm 3,7$ & $94,9 \pm 3,7$ & $97,2 \pm 3,4$ & $94,7 \pm 3,1$ \\
\hline Tarso & $21,3 \pm 0,8$ & $21,3 \pm 0,6$ & $21,6 \pm 0,6$ & $21,5 \pm 0,6$ \\
\hline Cráneo+Pico & $32,6 \pm 0,7$ & $32,4 \pm 0,4$ & $32,9 \pm 0,7$ & $32,6 \pm 0,6$ \\
\hline
\end{tabular}

Tabla 1.- Morfología de la lavandera cascadeña en Gipuzkoa. Para cada variable se indica el valor medio \pm desviación estándar (SD) y el tamaño muestral (n). Salvo el apuntamiento del ala, toda medida está en $\mathrm{mm}$. El periodo "verano" incluye los meses de abril a septiembre, mientras que el "invierno" abarca desde octubre hasta marzo.

Table 1.- Morphology of grey wagtails in Gipuzkoa. For each variable we show the mean \pm standard deviations (SD) and the sample size (n). Except for wing pointedness, all measurements are in $\mathrm{mm}$. The "summer" period includes the months of April to September, whilst "winter" spans the period from October to March.

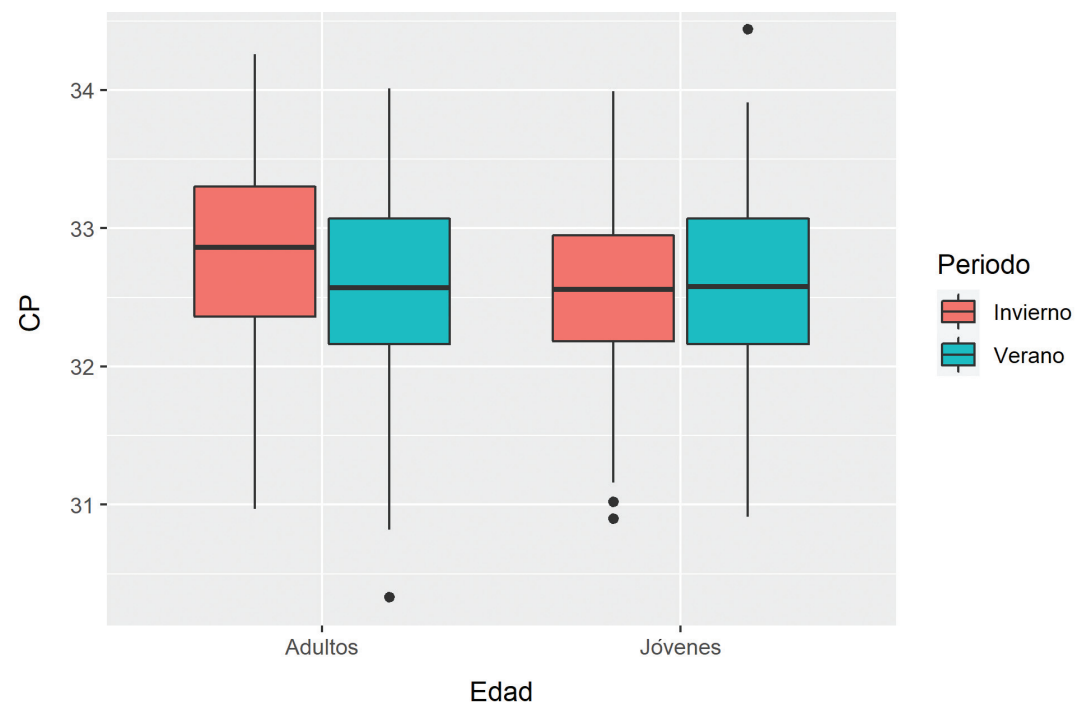

Fig. 1.- Diagrama de cajas donde se representa la longitud de cráneo + pico (CP) en función del periodo y la edad (adultos y jóvenes).

Fig. 1.- Boxplots of head + bill length (CP) in relation to period and age. 
caso de la variable cráneo+pico hay que considerar una interacción con la edad (Tabla 2). Así, la longitud alar en verano fue menor que en invierno (Tabla 1). En el caso de la variable cráneo+pico, sólo en el caso de los adultos hay que destacar un ligero incremento de la media de la variable en invierno, respecto al verano (Fig. 1).

En cuanto a la comparativa con otras zonas (Tabla 4), sólo se pudo llevar a cabo un análisis con aves del centro-occidente de Europa, dado que para el resto de regiones se juntaron aves de diferentes edades y la edad tiene efecto sobre la morfología de variables como la longitud alar y cola (Tabla 2). Así, las lavanderas del centro-occidente de Europa presentan alas más largas que las de sus congéneres capturados en Gipuzkoa, tanto en verano como en invierno, excepto machos jóvenes, para los que las diferencias no fueron significativas (Tabla 4). En el caso de la cola, para las aves de Gipuzkoa ésta tendió a ser más corta que en las lavanderas más nórdicas en adultos, si bien la diferencia sólo llegó al nivel de la significación en machos en verano

\begin{tabular}{|lccccc|}
\hline Factores & $L A$ & $L C$ & $L T$ & $C P$ & $P P$ \\
\hline Sexo & $F=118,70$ & $F=19,19$ & $F=1,22$ & $F=20,24$ & $F=8,28$ \\
& $P<0,001$ & $P<0,001$ & $P=0,271$ & $P<0,001$ & $P=0,004$ \\
\hline Edad & $F=35,65$ & $F=1,65$ & $F=3,97$ & $F=3,90$ & $F=2,98$ \\
& $P<0,001$ & $P=0,200$ & $P=0,047$ & $P=0,049$ & $P=0,085$ \\
\hline Periodo & $F=4,26$ & $F=2,06$ & $F=2,57$ & $F=1,82$ & $F=0,88$ \\
& $P=0,040$ & $P=0,152$ & $P=0,110$ & $P=0,178$ & $P=0,350$ \\
Sexo $\times$ Edad & $F=1,20$ & $F=0,50$ & $F<0,01$ & $F=0,11$ & $F=0,03$ \\
& $P=0,275$ & $P=0,479$ & $P=0,997$ & $P=0,742$ & $P=0,857$ \\
Sexo $\times$ Periodo & $F=1,41$ & $F=1,81$ & $F<0,01$ & $F=0,14$ & $F=0,08$ \\
& $P=0,236$ & $P=0,179$ & $P=0,960$ & $P=0,712$ & $P=0,777$ \\
Edad $\times$ Periodo & $F=1,21$ & $F=0,15$ & $F=3,70$ & $F=6,08$ & $F=0,29$ \\
& $P=0,272$ & $P=0,704$ & $P=0,055$ & $P=0,014$ & $P=0,590$ \\
\hline Sexo $\times$ Edad $\times$ Periodo & $F=0,42$ & $F=0,02$ & $F=0,67$ & $F=1,12$ & $F=0,12$ \\
& $P=0,518$ & $P=0,896$ & $P=0,415$ & $P=0,290$ & $P=0,726$ \\
\hline
\end{tabular}

Tabla 2.- ANOVA de $\mathrm{K}$ muestras realizado para determinar la existencia de diferencias significativas en la población de lavandera cascadeña en Gipuzkoa, según sexos, edades y periodos. Estadísticos: $F, F$ de Fisher; $P$, significación ligada al valor de $F(P$ es significativo si es inferior a 0,05$)$. Grados de libertad: edad, 1; sexo, 1; periodo, 1; residuales, 420. Variables: LA, longitud alar; LC, longitud de la cola; LT, longitud tarsal; CP, longitud de cráneo+pico; PP, apuntamiento del ala (para más detalles ver el índice $M$ descrito en la metodología).

Table 2.- K-samples ANOVA conducted to determine the effect of age, sex and period on morphology. Statistics: $F$, Fisher's $F$; $P$, level of significance. Degrees of freedom: age, 1; sex, 1; period, 1; residuals; 420. Variables: LA, wing length; $L C$, tail length; $L T$, tarsus length; $C P$, head+bill length; PP, wing pointedness (for details see $M$ index in Methods). 


\begin{tabular}{|lc|}
\hline Longitud alar & $\mathrm{HJ}^{\mathrm{a}}, \mathrm{HA}^{\mathrm{a}}, \mathrm{MJ}^{\mathrm{b}}, \mathrm{MA}^{\mathrm{c}}$ \\
\hline Longitud de la cola & $\mathrm{HJ}^{\mathrm{a}}, \mathrm{HA}^{\mathrm{a}}, \mathrm{MJ}^{\mathrm{ab}}, \mathrm{M}^{\mathrm{ab}}$ \\
\hline Longitud tarsal & $\mathrm{HJ}^{\mathrm{a}}, \mathrm{HA}^{\mathrm{a}}, \mathrm{MJ}^{\mathrm{a}}, \mathrm{MA}^{\mathrm{a}}$ \\
\hline Longitud de cráneo + pico & $\mathrm{HJ}^{\mathrm{a}}, \mathrm{HA}^{\mathrm{ab}}, \mathrm{MJ}^{\mathrm{ab}}, \mathrm{M}^{\mathrm{ab}}$ \\
\hline Apuntamiento alar & $\mathrm{HJ}^{\mathrm{a}}, \mathrm{HA}^{\mathrm{ab}}, \mathrm{MJ}^{\mathrm{ab}}, \mathrm{M}^{\mathrm{ab}}$ \\
\hline
\end{tabular}

Tabla 3.- Resultados de un test a posteriori de Tukey HSD realizado para determinar la existencia de diferencias entre sexos y edades. Los superíndices se utilizan para agrupar aquellas categorías de edad y sexo para las que no hubo diferencias significativas. Abreviaciones: $\mathrm{M}$, machos; $\mathrm{H}$, hembras; J, jóvenes; $A$, adultos.

Table 3.- Results from an a posteriori Tukey HSD test used to determine the existence of significant differences between the age and sex classes. Superscripts group those age and sex categories for which there were no significant differences. Abbreviations: $\mathrm{M}$, male; $\mathrm{H}$, female; J, young; $\mathrm{A}$, adult.

\begin{tabular}{|l|c|c|c|c|}
\hline & \multicolumn{2}{|c|}{ Jóvenes } & \multicolumn{2}{c|}{ Adultos } \\
\hline & $\sigma$ & $q$ & $\sigma$ & $q$ \\
\hline Longitud alar & $\begin{array}{c}83,6 \pm 1,6 \\
(25)\end{array}$ & $\begin{array}{c}82,7 \pm 1,8 \\
(16)\end{array}$ & $\begin{array}{c}85,0 \pm 2,0 \\
(13)\end{array}$ & $\begin{array}{c}83,8 \pm 2,1 \\
(10)\end{array}$ \\
\hline $\begin{array}{l}\text { Test de } t(\mathrm{P}) \\
\text { Verano }\end{array}$ & $\begin{array}{c}t=1,38 \\
(\mathrm{P}=0,174)\end{array}$ & $\begin{array}{c}t=4,49 \\
(\mathrm{P}<0,001)\end{array}$ & $\begin{array}{c}t=3,19 \\
(\mathrm{P}=0,002)\end{array}$ & $\begin{array}{c}t=5,91 \\
(\mathrm{P}<0,001)\end{array}$ \\
\hline $\begin{array}{l}\text { Test de } t(\mathrm{P}) \\
\text { Invierno }\end{array}$ & $\begin{array}{c}t=0,55 \\
(\mathrm{P}=0,581)\end{array}$ & $\begin{array}{c}t=5,63 \\
(\mathrm{P}<0,001)\end{array}$ & $\begin{array}{c}t=2,49 \\
(\mathrm{P}=0,014)\end{array}$ & $\begin{array}{c}t=3,66 \\
(\mathrm{P}=0,002)\end{array}$ \\
\hline Cola & $94,7 \pm 3,0$ & $\begin{array}{c}93,9 \pm 2,4 \\
(14)\end{array}$ & $\begin{array}{c}97,9 \pm 3,6 \\
(14)\end{array}$ & $\begin{array}{c}95,8 \pm 2,9 \\
(10)\end{array}$ \\
\hline $\begin{array}{l}\text { Test de } t(\mathrm{P}) \\
\text { Verano }\end{array}$ & $\begin{array}{c}t=1,85 \\
(\mathrm{P}=0,070)\end{array}$ & $\begin{array}{c}t=2,02 \\
(\mathrm{P}=0,049)\end{array}$ & $\begin{array}{c}t=2,98 \\
(\mathrm{P}=0,004)\end{array}$ & $\begin{array}{c}t=1,24 \\
(\mathrm{P}=0,221)\end{array}$ \\
\hline $\begin{array}{l}\text { Test de } t(\mathrm{P}) \\
\text { Invierno }\end{array}$ & $\begin{array}{c}t=4,52 \\
(\mathrm{P}<0,001)\end{array}$ & $\begin{array}{c}t=1,28 \\
(\mathrm{P}=0,214)\end{array}$ & $\begin{array}{c}t=1,77 \\
(\mathrm{P}=0,081)\end{array}$ & $\begin{array}{c}t=1,64 \\
(\mathrm{P}=0,116)\end{array}$ \\
\hline
\end{tabular}

Tabla 4.- Medias ( \pm desviación estándar $-S D$ - y tamaño muestral en paréntesis) de la longitud alar y de la cola de la lavandera cascadeña en otras zonas de Europa. Se indica, además, los resultados del test de $t$ (con su $P$ asociada) al comparar las medidas con los valores de referencia de Gipuzkoa mostrados en la Tabla 1 en verano y en invierno.

Table 4.- Mean ( \pm SD, sample size in parenthesis) wing and tail length of grey wagtails from other European areas. In addition, we show results from a one-sample $t$-test used to compare the measured values with the ones recorded in Gipuzkoa (Table 1), both in summer and winter. 
(y, marginalmente, en invierno; Tabla 4). En jóvenes, la cola tendió a ser más larga en aves de Gipuzkoa y, este caso, sólo significativamente en machos en invierno (y, marginalmente, en verano y también en hembras en verano; Tabla 4).

En cuanto a la fracción de aves jóvenes se detectó un efecto significativo del sexo en tres del total de cinco variables: longitud del ala, cola y cráneo+pico ( $P>0,05)$. $\mathrm{Al}$ incluir estas variables en el $A D$ se obtuvo la siguiente ecuación (valores en $\mathrm{mm}$ ): $Y=0,72$ (cráneo+pico) + 0,50 (longitud del ala) - 0.04 (longitud de la cola). Al aplicar esta fórmula, si $Y>-0,925$, entonces el ejemplar es macho y, si no, hembra. El porcentaje global de aves clasificadas correctamente es 69,0\% (machos: 91,3\%; hembras: $42,1 \%$ ). Repitiendo el AD para las aves capturadas en verano, el porcentaje de aves clasificadas correctamente no mejoró (67\%).

\section{Discusión}

Este es el primer artículo que describe la morfología de la lavandera cascadeña en la provincia de Gipuzkoa y, asimismo, en la región cantábrica y el conjunto de España, salvo islas Canarias. Para Canarias, no obstante, la literatura no diferencia aves adultas y jóvenes y, dado el peso de la edad en la morfología, no consideramos oportuno comparar la población de Gipuzkoa con las muestras que se han publicado para Canarias.

En general, y en lo relativo a la variabilidad de la morfología según sexos o edades, obtenemos resultados similares a trabajos previos, tanto de ésta como de otras especies de paseriformes: los machos son más grandes que las hembras y los adultos son más grandes que los jóvenes. Aunque cada medida mostró su propio patrón de variación, en la mayoría de los casos los valores máximos se registraron en machos adultos, y los mínimos, en hembras jóvenes.

En cuanto a la comparativa con poblaciones de otras zonas, la literatura disponible ofrece gran número de muestras, incluyendo otras subespecies tales como patriciae (Azores), schmitzi (Madeira), canariensis (Canarias), melanope (Asia central), robusta (Asia oriental), así como de la subespecie nominal cinerea. No obstante, en todos estos casos no se tuvo en cuenta la edad. Dado el conocido efecto de la edad en variables como la longitud alar o la cola, sólo se pudo comparar la población de Gipuzkoa con aquellas muestras que sí consideraban este factor separadamente. Esto redujo las comparativas que se llevaron a cabo en la Tabla 3 a muestras de Europa occidental, procedentes de pieles de museos (en Leiden y Amsterdam). En este contexto, la comparativa es deficiente y sería de mucho interés mejorar este aspecto para, como mínimo, el conjunto de Europa. Además, el hecho de comparar mediciones en pieles y en aves vivas también puede añadir un sesgo en el análisis, ya que las pieles suelen medir 
algo menos que las aves en vivo. Conscientes de este problema, dada la ausencia de alternativas consideramos oportuno llevar a cabo el análisis aquí descrito. Observamos, en todo caso, que las aves de Gipuzkoa son más pequeñas que las centroeuropeas en cuanto a longitud alar y cola (en este último caso sólo en adultos), pero al contrario en longitud de cola en aves jóvenes (más largas que las de Centroeuropa). En el caso del ala, la diferencia se ve aún más acentuada cuando se seleccionaron aves capturadas en Gipuzkoa durante un periodo en que la presencia de aves de origen extranjero es nula o marginal, entre los meses de abril a septiembre. En el caso de la cola, no obstante, estas diferencias entre periodos no son tan claras. Existe, en consecuencia, todo un campo de especulación respecto al porqué de todos estos resultados. Si bien la morfología está condicionada por diversos factores, a menudo y por la ley de Bergmann las poblaciones más nórdicas son más grandes como adaptación al frío, algo bien demostrado en aves. Si éste es el caso de la lavandera cascadeña es algo que deberá ser analizado en el futuro, considerando varias poblaciones y teniendo en cuenta otro tipo de factores. Por otro lado, debemos considerar también el efecto de la migración en la biometría. Así, las aves que migran tienen alas proporcionalmente más largas así como una cola comparativamente más corta que, en conjunto, mejoran la eficacia de vuelo. Esto podría concordar con los resultados hallados para la fracción de los jóvenes que, en Gipuzkoa, tienen alas más cortas así como cola más larga que las aves más nórdicas, sugiriendo para las aves de Gipuzkoa un comportamiento como mínimo más sedentario que las centroeuropeas. La bibliografía, así, describe que las lavanderas cascadeñas más nórdicas son más migratorias que las más meridionales y, además, este fenómeno es más común entre las aves jóvenes. Los adultos, sedentarios en buena parte de su área de distribución o con distancias más cortas de migración, no responderían quizás a este patrón geográfico revelado en las lavanderas más jóvenes. Así, las diferencias que observamos en adultos se corresponderían, principalmente, a variaciones en el tamaño. En todo caso, se trata de un tema que requiere ser analizado con más rigor y profundidad en el futuro. Finalmente, no debe obviarse el tamaño muestral, reducido, cuyo efecto sobre tales resultados debería, también, descartarse en nuevos estudios.

El análisis discriminante (AD) proporcionó una fórmula que sólo clasificó bien el 68\% de la muestra de jóvenes lavanderas cascadeñas. Esto es debido a la existencia de un alto solapamiento biométrico entre los sexos, dentro de esta edad, lo que en la práctica supone la no recomendación del uso de la ecuación dada su baja fiabilidad. Para recomendarla, el poder de esta ecuación debería alcanzar $>90 \%$ de la muestra. Un motivo por el que un AD puede dar lugar a una ecuación con poco poder discriminante puede ser un tamaño muestral bajo. No obstante, el tamaño muestral en este caso es razonablemente alto $(n=259)$, por lo que, a priori, descartamos esta opción como motivo del escaso poder discriminante. Otra opción es que las muestras usadas para este análisis fueran heterogéneas (esto es, que tuvieran una varianza 
asociada alta), en la medida en que se trata de aves capturadas en diferentes años y a lo largo de varios meses dentro de un mismo ciclo anual. Los efectos del desgaste y factores adicionales como la presencia de aves foráneas en determinadas épocas pudo también afectar al AD. Finalmente, no hay que descartar que, en muchas especies, el alto solapamiento biométrico entre sexos no permite su diferenciación a partir de la morfología.

\section{Agradecimientos}

Este estudio fue parcialmente financiado por la Diputación de Gipuzkoa y el Gobierno Vasco. La Diputación de Gipuzkoa autorizó el anillamiento de aves. J. I. Aguirre y D. Álvarez proporcionaron valiosos comentarios que nos ayudaron a mejorar una primera versión de este artículo.

\section{Bibliografía}

Aierbe, T., Olano, M., Vázquez, J., 2001. Atlas de las aves nidificantes de Gipuzkoa. Munibe, Cienc. nat., 52.

Alonso, D., Arizaga, J., 2005. Efecto de la edad, el sexo y el tiempo en la biometría del piquituerto común (Loxia curvirostra curvirostra) en Navarra. Munibe, Cienc. Nat. 56, 133 144.

Alonso, D., Arizaga, J., 2006. Biometrics of Citril Finch Serinus citrinella in the West Pyrennes and the influence of feather abrasion on biometric data. Ringing \& Migration 23, 116-124.

Alonso, D., Arizaga, J., Miranda, R., Hernandez, M.A., 2006. Morphological diversification of common crossbill Loxia curvirostra populations within Iberia and the Balearics. Ardea 94, 99-107.

Arizaga, J., Andueza, M., Fernández, B., 2017. Morfología de aves paseriformes nidificantes en Navarra y regiones limítrofes. Sociedad de Ciencias Aranzadi, Donostia.

Arizaga, J., Campos, F., Alonso, D., 2006. Variations in wing morphology among subspecies might reflect different migration distances in Bluethroat. Ornis Fennica 83, 162-169.

Arizaga, J., Hernandez, M.A., Rivas, J., Miranda, R., 2009. Biometrics of Iberian Dippers Cinclus cinclus: environmental sources of among-population variation. Ardea 97, 23-30.

Arzak, A., Jauregi, J.I., Goikoetxea, J., Sánchez, J.M., Aranguren, I., Arizaga, J., 2014. Sexado de Mirlos acuáticos Cinclus cinclus en Gipuzkoa a partir de la biometría. Revista Catalana d’Ornitología 30, 54-62.

Ashton, K.G., 2002. Patterns of within-species body size variation of birds: strong evidence for Bergmann's rule. Global Ecology and Biogeography 11, 505-523. 
Calmaestra, R., Moreno, E., 2001. A phylogenetically-based analysis on the relationship between wing morphology and migratory behaviour in passeriformes. Ardea 89, 407-416.

Cramp, S., 1988. Handbook of the Birds of Europe, the Middle East and North Africa. Vol. 5. Oxford University Press, Oxford.

Cristol, D.A., Baker, M.B., Carbone, C., 1999. Differential migration revisited. Latitudinal segregation by age and sex class. In: Nolan, V.J., Ketterson, E.D., Thompson, C.F. (Eds.), Current Ornithology, 33-88. Academic Press, New York.

Delingat, J., Hobson, K., Dierschke, V., Schmaljohann, H., Bairlein, F., 2010. Morphometrics and stable isotopes differentiate populations of Northern Wheatears (Oenanthe oenanthe). Journal of Ornithology 152, 383-395.

Fiedler, W., 2005. Ecomorphology of the external flight apparatus of Blackcaps (Sylvia atricapilla) with different migration behavior. Annals of the New York Academy of Sciences 1046, 253-263.

Förschler, M.I., Bairlein, F., 2010. Morphological shifts of the external flight apparatus across the range of a passerine (Northern Wheatear) with diverging migratory behaviour. Plos One 6, e18732.

Jenni, L., Winkler, R., 1989. The feather-length of small passerines: a measurement for wing-length in live birds and museum skins. Bird Study 36, 1-15.

Jenni, L., Winkler, R., 1994. Moult and ageing of European passerines. Academic Press, London.

López, V., 2003. Lavandera Cascadeña Motacilla cinerea. In: Martí, R., Del Moral, J.C. (Eds.), Atlas de las aves reproductoras de España, 400-401. SEO/BirdLife-MMA, Madrid.

Margalef, R., 1998. Ecología. Omega, Barcelona.

Mönkkönen, M., 1995. Do migrant birds have more pointed wings?: a comparative study. Evolutionary Ecology 9, 520-528.

Pérez-Tris, J., Asensio, B., 1997. Migración e invernada de las lavanderas cascadeña Motacilla cinerea y blanca M. alba en la península ibérica e islas Baleares. Doñana Acta Vertebrata 24, 79-89.

R Core Team, 2014. R: A language and environment for statistical computing. Vienna, Austria.

Salewski, V., Hochachka, W.M., Fiedler, W., 2010. Global warming and Bergmann's rule: do central European passerines adjust their body size to rising temperatures? Oecologia $162,247-260$.

Svensson, L., 1996. Guía para la identificación de los paseriformes europeos. Sociedad Española de Ornitología, Madrid.

Tellería, J.L., Carbonell, R., 1999. Morphometric variation of five Iberian Blackcap Sylvia atricapilla populations. Journal of Avian Biology 30, 63-71. 
Venables, W.N., Ripley, B.D. 2002. Modern Applied Statistics with S (4th edition). Springer, New York.

Yom-Tov, Y., Yom-Tov, S., Wright, J., J. R. Thorne, C., Du Feu, R., 2006. Recent changes in body weight and wing length among some British passerine birds. Oikos 112, 91-101.

Fecha de recepcion/Date of reception: 10/07/2019 Fecha de aceptacion/Date of acceptance: 10/04/2020 Editor Asociado/Associate editor: David Álvarez 